\title{
Estimación del diámetro, altura y volumen a partir del diámetro del tocón para Quercus laurina, en Ixtlán, Oaxaca, México
}

\author{
Estimation of diameter, height and volume from the stump \\ diameter for Quercus laurina, at Ixtlan, Oaxaca, Mexico
}

Joel Martínez-López’ y Alejandra Acosta-Ramos’

1 Instituto de Estudios Ambientales. Universidad de la

Sierra Juárez. Oaxaca, México.

mjoel@juppa.unsij.edu.mx

\section{RESUMEN}

En el presente trabajo se generaron tres ecuaciones para estimar el diámetro normal, altura y volumen total a partir del diámetro del tocón para Quercus laurina en el predio forestal de Ixtlán de Juárez, Oax., con una muestra de 54 árboles. Para obtener la ecuación más confiable a la relación funcional: (a) diámetro normal-diámetro del tocón, (b) altura total-diámetro del tocón y (c) volumen totaldiámetro del tocón, se ajustaron modelos lineales y no lineales a la base de datos. Los criterios para definir el ajuste en los tres casos se basó en el coeficiente de determinación normal y ajustado, el cuadrado medio del error, P-valor, coeficiente de variación, suma y dispersión de residuales contra valores predichos. El modelo con mayor bondad de ajuste para la estimación del diámetro normal con base en el diámetro del tocón fue el modelo de regresión lineal simple, y para los otros dos casos, fue el modelo potencial. Con las correspondientes ecuaciones generadas se pueden estimar de forma precisa los valores de las variables dependientes y evaluar diferentes escenarios en áreas afectadas por fenómenos naturales o antropogénicos.

PALABRAS CLAVE: Estimador, homogeneidad de varianzas, ecuaciones de predicción, modelos lineales, modelos no lineales.

\section{ABSTRACT}

In this paper, three equations were generated to estimate the normal diameter, height and total volume from stump diameter for Quercus laurina stands in Ixtlan de Juarez, Oaxaca, with a sample of 54 trees. Linear and nonlinear models were adjusted to the database to obtain the most reliable equation to the functional relationship: (a) normal diameter - stump diameter, (b) total height - stump diameter and (c) total volume-stump diameter. The criteria for defining the adjustment in all three cases was based on: normal and adjusted coefficient of determination, mean square error, P-value, coefficient of variation, and sum and dispersion of residuals against predicted values. The model with the highest goodness of fit for the estimated normal diameter based on the diameter of the stump was the simple linear regression model, and in the other two cases, it was the potential model. With these equations, it is possible to accurately estimate the dependent variables values and evaluate different scenarios in areas affected by natural or anthropogenic phenomena.

KEY WORDS: Estimator, variance homogeneity, prediction equations, linear models, non-linear models. 


\section{INTRODUCCIÓN}

El aprovechamiento maderable ha tenido gran relevancia para la sociedad debido a sus diversos beneficios. Interesa por lo tanto tener una estimación precisa de las existencias volumétricas que permita la planificación del aprovechamiento sustentable. Sin embargo, en ocasiones el bosque es afectado por diversos fenómenos que dejan sólo como evidencia el tocón de los árboles.

No obstante, esta evidencia permite la reconstrucción del tamaño de los árboles removidos y es tal su importancia que resulta de utilidad para revisar volúmenes de cosecha, venta de madera basada en diámetro del tocón, valorar daños causados por eventos catastróficos, registros históricos de actividades de manejo, establecimiento de pérdidas por aprovechamientos clandestinos y el valor de los árboles (McClure, 1968; Wharton, 1984; Schlieter, 1986; Shrivastava y Singh, 2003; Corral-Rivas, 2007; Westfall, 2010). Las dimensiones del tocón se emplean también para conocer el diámetro basal original, la distribución de las especies de los individuos, y datos para la determinación de la distribución de las clases de tamaños de los árboles del rodal; los tocones preservan características que permiten la determinación de especies extraídas y la anatomía de la madera (Williams et al., 2003).

Es posible determinar la relación funcional del diámetro del tocón con algunas variables dasométricas como el diámetro normal, altura o volumen, haciendo uso de la regresión, lineal o no lineal, o con el apoyo de tablas preparadas para tal efecto (Bylin, 1982; Westfall, 2010). El tocón puede servir como referencia cuando las mediciones del diámetro normal y la altura del árbol no se pueden realizar. Consecuentemente, la tabla de volumen o ecuaciones basadas en el diámetro a la altura del pecho no se pueden utilizar directamente (Aigbe et al., 2012), sino que éstas deben estimarse de forma indirecta a través de ecuaciones ajustadas para ello.

Entre los primeros esfuerzos para predecir el área basal y el diámetro a la altura del pecho empleando mediciones del tocón, se encuentran los trabajos de McClure (1968) que utilizó una transformación logarítmica para desarrollar un modelo de regresión lineal para 53 especies;
Bylin (1982) generó ecuaciones de predicción del volumen empleando diámetro del tronco y la altura del tocón como variables independientes; Wharton (1984) determinó relaciones entre el diámetro del tocón y el diámetro a la altura del pecho para 17 especies; Schlieter (1986) generó modelos de predicción del diámetro a la altura del pecho por medio de mínimos cuadrados ordinarios y regresión lineal simple para Pinus contorta; todos estos trabajos realizados en Estados Unidos de Norteamérica.

En India, Shrivastava y Singh (2003) determinaron interrelaciones entre el diámetro a la altura del pecho y el diámetro del tronco en Abies pindrow Spach.; en el ártico de Canadá, Williams et al. (2003) emplearon un método para la reconstrucción de biomasa, estructura y productividad de árboles para un bosque terciario del género Metasequoia a partir de las dimensiones del tocón; Diéguez et al. (2003), estimaron el diámetro a la altura del pecho y el volumen para 6 especies forestales en España; Benítez et al., (2004) estimaron el diámetro a la altura del pecho en plantaciones de Casuarina equisetifolia Forst, en Cuba. En México Corral-Rivas et al. (2007) desarrollaron modelos lineales y no lineales de predicción del diámetro a la altura del pecho y el volumen para 5 coníferas en El Salto, Durango. Ozcelik et al. (2010), desarrollaron ecuaciones de predicción del diámetro y el volumen usando un modelo lineal simple para especies importantes en Turquía; Westfall, (2010) determinó nuevos modelos de predicción del diámetro a la altura del pecho empleando un enfoque de modelo de efectos mixtos.

Recientemente, en México Pompa-García et al. (2011) determinaron modelos dendrométricos para estimación del diámetro a la altura del pecho para Pinus durangensis; Aigbe et al. (2012) desarrollaron un modelo de predicción del volumen para plantaciones de Terminalia ivorensis en Nigeria; y Senyurt (2012) generó ecuaciones para conocer la relación del diámetro a la altura del pecho y diámetro del tocón por medio de modelos de regresión, para la región occidental del Mar Negro en Turquía.

Es notable que en la última década se hayan incrementado la cantidad de trabajos en predicción del diámetro a la altura del pecho a partir de las dimensiones del 
tocón. No obstante para México, la cantidad de esfuerzos es muy limitada y se ha concentrado principalmente en coníferas, por tanto es imperativo contar con este tipo de herramientas basadas en procedimientos estadísticos rigurosos que permitan estimar volúmenes comerciales y totales en el grupo taxonómico de encinos, que es uno de los más numerosos e importantes en nuestro país (PompaGarcía y Solís-Moreno, 2008).

Los encinos en México se encuentran representados ampliamente por el género Quercus con aproximadamente 160 especies. En Oaxaca se han descrito 52 especies de éstos ubicándose en el tercer lugar a nivel nacional en diversidad de encinos. De acuerdo con Valencia y Nixon (2004), Ixtlán es uno de los distritos con mayor riqueza de encinos, y Quercus laurina posee la distribución más amplia. La madera de los encinos está considerada como una de las de mayor calidad; es altamente valorada e importante en la economía europea (Valencia y Nixon, 2004); sin embargo, aunque en México la producción maderable de encinos es baja, su alta diversidad y amplia distribución podrían incrementar el potencial de producción maderera y contribuir en la economía regional.

\section{Objetivo}

El objetivo del presente trabajo consistió en determinar la relación funcional del diámetro normal, altura y volumen respecto al diámetro del tocón, para Quercus laurina en Ixtlán de Juárez, Oaxaca.

\section{MATERIALES Y MÉTODOS}

\section{Área de estudio}

Ixtlán de Juárez, se ubica en la Sierra Norte del estado de Oaxaca, a una distancia aproximada de 61 kilómetros de la capital. El predio comunal abarca una superficie de 19180 ha en donde se reconocen los siguientes tipos de vegetación: bosque de pino-encino, bosque de encino-pino, bosque mesófilo de montaña, bosque de encino, selva baja caducifolia y vegetación secundaria derivada del bosque de pinoencino. El predio bajo aprovechamiento forestal se localiza dentro de las siguientes coordenadas geográficas: $17^{\circ} 18^{\prime}$
16 " y $17^{\circ} 30^{\prime} 00^{\prime \prime}$ Latitud y $96^{\circ} 21^{\prime} 29$ " y $96^{\circ} 31^{\prime} 38^{\prime}$ "Longitud (TIASA, 2003). Las especies de encino presentes en el predio forestal son: Quercus crassifolia, Quercus rugosa y Quercus laurina. Las especies de importancia maderable que se distribuyen dentro del predio comunal son: Pinus oaxacana Mirov, P. patula Schl. et Cham., P. patula var. longepedunculata Look, $P$. pseudostrobus Lindl., P. rudis Endl., P. douglasiana Martínez, P. teocote Schl et Cham., P. ayacahuite Schl. y P. leiophylla Schl et Cham.

\section{Árboles de interés}

En el área de estudio se recolectaron datos de 54 árboles de Quercus laurina ubicados de forma selectiva y derribados en las áreas de corta correspondientes a las anualidades 2009 y 2010 indicadas en el Programa de Manejo Forestal. Se seleccionaron árboles con diferente diámetro y altura, que cumplieran con las características siguientes: copa completa, no despuntados, no rayados, sin evidencia de plagas y enfermedades, no podridos ni huecos, y de porte saludable. Se procuró seleccionar árboles de un sólo fuste para evitar la sobreestimación de la población. Para tener una base de datos completa y cubrir todas las categorías de diámetros y alturas existentes, se midieron de 3 a 5 árboles por categoría, aunque en ocasiones se utilizó una muestra mayor dependiendo de su existencia. Los árboles fueron elegidos en las diferentes áreas donde se distribuye la especie para obtener una base de datos representativa del área forestal, así mismo, por las diferentes condiciones ambientales y ecológicas presentes en el predio comunal.

\section{Variables medidas}

En cada árbol se midió la altura total $(A, \mathrm{~m})$ empleando cinta métrica y el diámetro normal con corteza $(D n, \mathrm{~cm})$, en la parte superior de la ladera, empleando cinta diamétrica. El diámetro del tocón $(D t, \mathrm{~cm})$ se midió con cinta diamétrica y se consideró como el diámetro extremo superior de la parte del tronco que queda en pie tras la corta. Al árbol derribado y seccionado se le midió el diámetro en la parte media y en los extremos, además de la longitud de trozas y ramas para obtener su volumen individual 
empleando la fórmula de Huber o Smalian según fuese el caso y, finalmente, se obtuvo el volumen por árbol $\left(V, \mathrm{~m}^{3}\right)$ con la sumatoria de sus partes. El diámetro del tocón, diámetro normal y volumen total incluye el grosor de corteza en el presente trabajo, para conocer el volumen sin corteza se recomienda reducir en $23 \%$ de volumen rollo total árbol de acuerdo con Pacheco (2011).

\section{Ajuste de modelos}

Para determinar el modelo con mayor bondad de ajuste de las relaciones funcionales: a) diámetro normal-diámetro del tocón, b) altura total-diámetro del tocón, y c) volumen-diámetro del tocón, los datos se procesaron empleando el paquete estadístico SAS versión 9.0 (SAS Institute Inc., 2002).

Previo al ajuste de modelos se analizó la gráfica de dispersión para conocer la tendencia de distribución de la variable dependiente respecto a la independiente. Posteriormente, se ajustó para los incisos (a) y (b), un modelo lineal, un polinomial y un alométrico, potencial o multiplicativo (Avery y Burkhart, 2002; Gutiérrez y De la Vara, 2008; Vanclay, 1994). Para el caso del inciso (c), se ajustó el modelo potencial y de Schumacher (Avery y Burkhart, 2002; Husch et al., 2003). Los modelos empleados para las relaciones funcionales requeridas se muestran en la tabla 1.

En el ajuste de los datos de las relaciones funcionales $D n-D t$ y A-D $t$, los datos se procesaron empleando la ins- trucción GLM del programa estadístico y NLIN METHOD=DUD mediante el procedimiento de mínimos cuadrados, en el que es común verificar problemas de heterocedasticidad (Cruz et al., 2008). Para corroborar el supuesto de normalidad se obtuvieron los coeficientes de asimetría y curtosis para los valores predichos de cada modelo, empleando el procedimiento UNIVARIATE de SAS.

Con respecto al ajuste de la relación funcional $V-D t$, los datos se procesaron por medio del procedimiento NLIN METHOD=DUD, del programa indicado con anterioridad, mediante el método de mínimos cuadrados. Para corroborar el supuesto de normalidad, se obtuvo el coeficiente de asimetría y curtosis para los valores predichos de cada modelo.

La capacidad de ajuste de las relaciones funcionales en todos los casos se analizó tomando como criterios de decisión los indicadores estadísticos sugeridos por Diéguez et al. (2003) y Pompa-García et al. (2011), como son: coeficiente de determinación normal $\left(\mathrm{R}^{2}\right)$ y ajustado $\left(\mathrm{R}^{2}\right.$ ajustado), que representa la parte de la varianza explicada por el modelo y además considera el numero de parámetros del modelo para el segundo caso, cuadrado medio del error (CME), que analiza la precisión de las estimaciones. Además se consideraron $\operatorname{Pr}>\mathrm{F}$, coeficiente de variación (CV), suma, y análisis gráfico de dispersión de residuales contra valores predichos, para detectar errores o comportamientos anormales.

TABLA 1. Expresiones matemáticas de los modelos seleccionados para el ajuste a los datos.

\begin{tabular}{ccc}
\hline Relación & \multicolumn{1}{c}{ Modelo } & Tipo de modelo \\
\hline Dn-Dt & $Y=\beta_{0}+\beta_{1} D t$ & Modelo lineal \\
\cline { 2 - 3 } & $Y=\beta_{0}+\beta_{1} D t+\beta_{2} D t^{2}$ & Modelo polinomial \\
\cline { 2 - 3 } A-D & $Y=\beta_{0} D t^{\beta 1}$ & Modelo alométrico (potencial o multiplicativo) \\
\hline$V-D t$ & $V=\beta_{0} D t^{\beta 1}$ & Modelo potencial \\
\cline { 2 - 3 } & $V=\beta_{0} e^{\beta 1 / D t}$ & Modelo de Schumacher \\
\hline
\end{tabular}

Donde: $Y=$ diámetro normal $(D n$ en $\mathrm{cm})$ o altura total $(A$ en $\mathrm{m}), D t=$ diámetro del tocón $(\mathrm{en} \mathrm{cm}), V=$ volumen total $\left(\right.$ en $\left.\mathrm{m}^{3}\right), \mathrm{y} \beta_{i}=$ parámetros del modelo. 


\section{RESULTADOS Y DISCUSIÓN}

Los principales valores estadísticos para las variables consideradas: diámetro del tocón $(D t)$, diámetro normal $(D n)$, altura $(A)$ y volumen $(V)$, se presentan en la tabla 2 . Las diferencias entre los valores mínimos y máximos de cada variable se ven reflejadas en la desviación estándar, coeficiente de variación, sesgo y coeficiente de curtosis. Sin embargo, al tratarse de un ajuste de modelos, la base de datos debe cubrir la mayoría de las categorías diamétricas y alturas existentes, por ello, aunque la desviación estándar es relativamente alta y, más estrictamente, el coeficiente de variación que se observa con valores altos, los datos son confiables para hacer el ajuste de modelos y predecir de forma significativa el diámetro normal, altura y volumen, a partir del diámetro del tocón. En el ambiente forestal se cree que el tocón debe tener una altura regular de $30 \mathrm{~cm}$, pero no siempre sucede así, como lo reconocen Jenkins et al. (2004), sobre todo en bosques de topografía accidentada, y debido a la diversidad de técnicas para el derribo.
Barrio et al. (2004), quienes elaboraron una tarifa con clasificación de productos basada en un modelo de volumen porcentual para Quercus robur L., en Galicia, España, trabajaron con una base de datos con un coeficiente de variación de $67,21 \%$ en el volumen e intervalo de 1,63 en sus valores. Para el presente trabajo, el intervalo es de 4,5025 con una variación de $120,45 \%$, lo que indica que a mayor diferencia entre ellos, las medidas de dispersión se incrementan proporcionalmente.

\section{Relación diámetro normal-diámetro del tocón (Dn-Dt)}

La dispersión de los valores para el diámetro normal respecto al diámetro del tocón mostró una tendencia lineal (Fig. 1a), por ello se considera que los modelos lineales (lineal y polinomial) podrían tener mayor bondad de ajuste comparado con el modelo potencial. Los indicadores estadísticos calculados para definir la bondad de ajuste se muestran en la tabla 3.

Tabla 2. Estadísticos descriptivos de los datos para Quercus laurina, en Ixtlán de Juárez, Oaxaca, México.

\begin{tabular}{lllllllll}
\hline Variable & $n$ & Mín. & Máx. & Media & STD & CV & Asimetría & Curtosis \\
\hline$D t$ & 54 & 7,5 & 72,00 & 34,6519 & 18,1443 & 52,36 & 0,337 & $-1,033$ \\
\hline$D n$ & 54 & 6,0 & 63,00 & 29,6315 & 16,5857 & 55,97 & 0,369 & $-1,038$ \\
\hline$A$ & 54 & 5,7 & 34,46 & 19,0819 & 8,6970 & 45,58 & 0,138 & $-1,260$ \\
\hline$V$ & 54 & 0,0121 & 4,5146 & 1,0659 & 1,2842 & 120,48 & 1,269 & 0,366 \\
\hline
\end{tabular}

n = tamaño de muestra, Mín = valor mínimo, Máx = valor máximo, STD = desviación estándar, CV = coeficiente de variación, Asimetría = coeficiente de asimetría y Curtosis = Coeficiente de curtosis.

TABLA 3. Indicadores estadísticos de los modelos ajustados para estimar el diámetro normal con base al diámetro del tocón para Quercus laurina, en Ixtlán de Juárez, Oaxaca, México.

\begin{tabular}{lllllll}
\hline Modelo & $R^{2}$ & $R^{2}$-adj & $C M E$ & Pr>F & $C V(\%)$ & Sum res \\
\hline Modelo lineal & 0,9870 & 0,9868 & 3,62 & $<0,0001$ & 6,42 & 0,0000 \\
\hline Modelo polinomial & 0,9871 & 0,9866 & 3,67 & $<0,0001$ & 6,46 & $-0,0000$ \\
\hline Modelo potencial & 0,9969 & 0,9968 & 3,61 & $<0,0001$ & 6,41 & $-1,1173$ \\
\hline
\end{tabular}

$R^{2}=$ Coeficiente de determinación normal, $R^{2}$-adj = Coeficiente de determinación ajustado, CME = Cuadrado Medio del Error, Pr>F = Probabilidad de $\mathrm{P}$ y Sum res = Suma de residuales. 
De acuerdo con estos valores estadísticos, todos los modelos mostraron una aceptable bondad de ajuste; sin embargo, los modelos lineales (lineal y polinomial) generaron una suma de residuales igual a cero y, de ellos, el modelo lineal obtuvo un cuadrado medio del error y coeficiente de variación más bajo. El coeficiente de determinación normal, que representa el porcentaje de la variable dependiente que es explicada por la independiente, en todos los casos es cercano a 1 ; y para el coeficiente de determinación ajustado, el cual es similar al normal, explica además el efecto de las diferentes variables independientes. En todos los modelos ajustados, los coeficientes de determinación son aceptables de acuerdo con Alder y Cailliez (1980), quienes expresan que las mejores funciones pueden tener coeficientes de 0,7 y 0,8; aunque Gujarati (2004) plantea que un modelo es satisfactorio si el valor de éste es alrededor de 0,8 .

Debido a la simplicidad del modelo lineal, considerando además su CME y CV, se concluye que es el modelo más adecuado para estimar el diámetro normal a partir del diámetro del tocón para Quercus laurina en el área de estudio. Para todos los modelos ajustados, el coeficiente de asimetría de los valores predichos osciló de 0,33 (modelo lineal) a 0,38 (modelo potencial), y el coeficiente de curtosis osciló de -1,03 (lineal) a -1,00 (potencial). El gráfico de residuales contra valores predichos del modelo lineal se muestra en el inciso b de la figura 1 , en el cual se observa que no existen problemas de heterocedasticidad.

No siempre los modelos más complejos son los que predicen de mejor forma la variable respuesta, como es el caso del trabajo de Barrio et al. (2004), en el que comprobaron que las ecuaciones que incluyen términos polinómicos o productos cruzados no aportan nada nuevo a la explicación de la variabilidad. Los resultados de esta investigación son similares al encontrado por Diéguez et al. (2003), en un trabajo con diversas especies forestales de España, y al de Corral-Rivas et al. (2007), quienes trabajaron con pináceas en el norte de México. El error estándar (EE), valor de $t$ y significancia de los parámetros del modelo de mayor bondad de ajuste se muestran en la tabla 4. Para el parámetro $\beta_{0}$ se rechaza la hipótesis nula que sostiene que el punto de intercepto con el eje de las abscisas es cero, algo similar ocurre para el parámetro $\beta_{1}$. Lo anterior indica que los parámetros del modelo ayudan a explicar de forma significativa la variable respuesta con una confiabilidad del $95 \%$.

\section{Relación altura-diámetro del tocón (A-Dt)}

El gráfico de dispersión para los datos de la altura total (A) respecto al diámetro del tocón $(D t)$ se muestra en la figura 2 (inciso a), donde se percibe que los valores se dis-
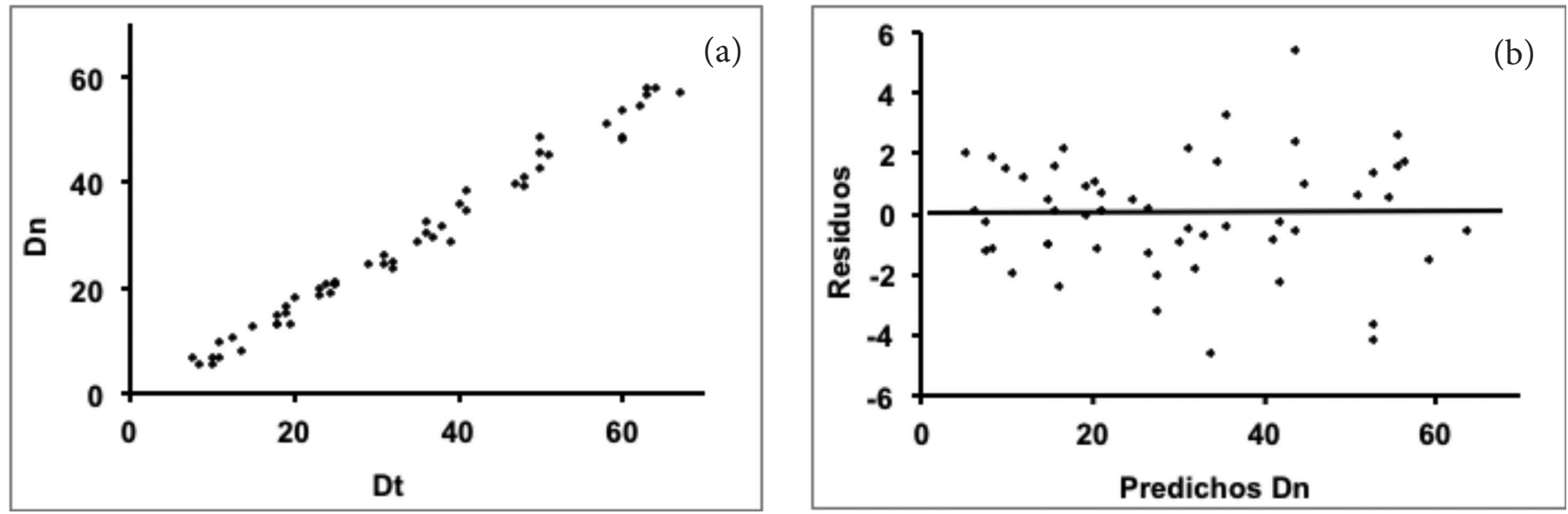

FIGURA 1. Dispersión de valores del diámetro normal contra diámetro del tocón (a) y dispersión de residuales contra valores predichos de diámetro normal (b), para Quercus laurina en Ixtlán de Juárez, Oaxaca, México. 
TABLA 4. Error estándar, valor de $t$ y significancia de los parámetros de ajuste del modelo lineal.

\begin{tabular}{cccccc}
\hline Modelo & Parámetro & Estimador & $E E$ & $t$ & Pr>t \\
\hline \multirow{2}{*}{ Modelo lineal } & $\beta_{0}$ & $-1,838555212$ & 0,56235791 & $-3,27$ & 0,0019 \\
\cline { 2 - 6 } & $\beta_{1}$ & 0,908177630 & 0,01440585 & 63,04 & $<0,0001$ \\
\hline
\end{tabular}
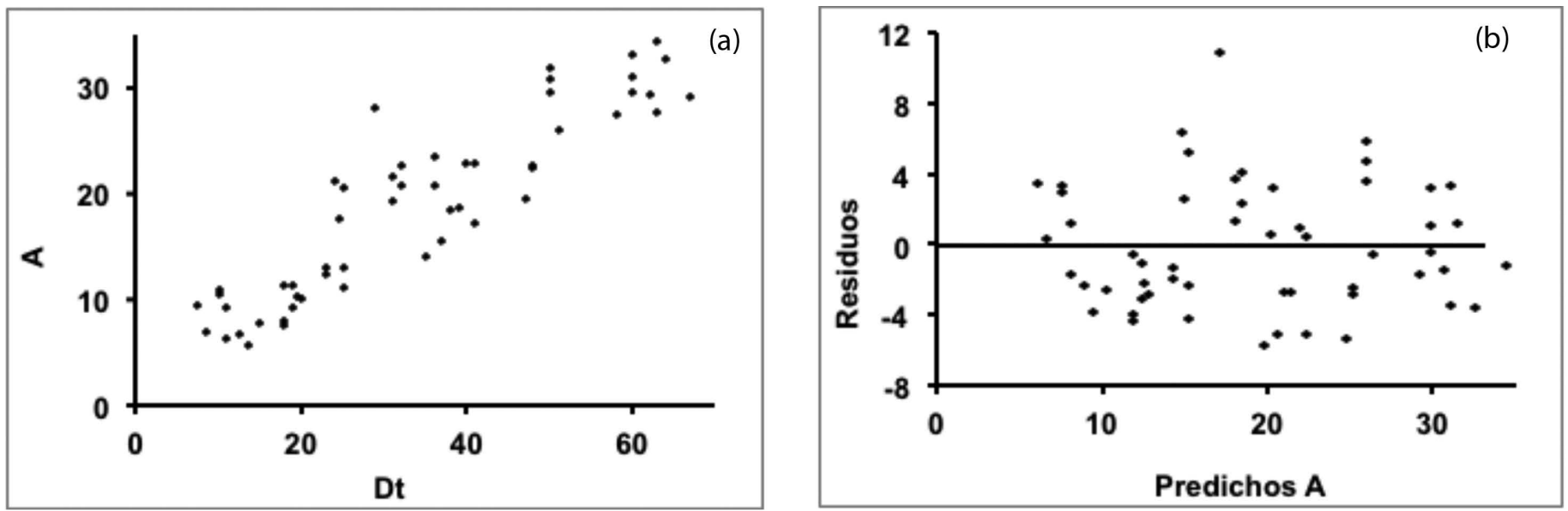

FIGURA 2. Dispersión de valores de la altura contra diámetro del tocón (a) y dispersión de residuales contra valores predichos de altura (b), para Quercus laurina en Ixtlán de Juárez, Oaxaca, México.

tribuyen con una tendencia lineal positiva. En la tabla 5, se concentran los indicadores estadísticos para definir la bondad de ajuste de los modelos analizados.

Todos los modelos se ajustaron favorablemente a la base de datos, mientras que el modelo potencial (alométrico) se ajustó de mejor manera debido a que su coeficiente de determinación normal y ajustado es superior al resto de los modelos y además generó un cuadrado medio del error y coeficiente de variación de menor valor. El coeficiente de asimetría y de curtosis para el modelo potencial es de 0,16 y -1,09, respectivamente. Derivado de lo anterior, se concluye que la relación funcional entre la altura y el diámetro del tocón es de tipo potencial.

Con respecto a la relación altura-diámetro del tocón, Diéguez et al. (2003) encontraron que la altura del tocón no aporta un incremento significativo sobre la variabilidad explicada por los modelos, solo recomiendan considerar la altura del tocón en el caso de especies con particularidades en la base del tronco. Por otra parte,
Martínez (2001) y Ruiz (2011) relacionaron la altura de árboles dominantes y codominantes respecto a su diámetro normal para Pinus patula Schl. et Cham., en la Sierra Norte del Estado de Oaxaca y encontraron que esta relación funcional puede ser estimada a través del Modelo de Schumacher (modelo sigmoidal). El diámetro normal y el diámetro del tocón puede ser modelado a través de regresión lineal simple, como se encontró en la presente investigación, por lo que la relación funcional entre la altura del árbol y el diámetro del tocón debe ser de tipo potencial.

Corral-Rivas et al. (2007) sugieren una mayor investigación para observar la variabilidad explicada por dicha variable cuando las cortas no se efectúan a los $30 \mathrm{~cm}$. Pompa-García y Solís-Moreno (2008), ajustaron ecuaciones para predecir el volumen en Quercus en Chihuahua, al norte de México y encontraron que el modelo polinomial de Rentería (1995) puede ser usado de forma confiable para predecir el diámetro a cualquier altura del trozo y la altura a cualquier diámetro del mismo, dicho modelo 
TABLA 5. Indicadores estadísticos de los modelos ajustados para estimar la altura en base al diámetro del tocón para Quercus laurina, en Ixtlán de Juárez, Oaxaca.

\begin{tabular}{lllllll}
\hline Modelo & $R^{2}$ & $R^{2}$-adj & $C M E$ & Pr $>F$ & $C V(\%)$ & Sum res \\
\hline Modelo lineal & 0,8332 & 0,8300 & 12,85 & $<0,0001$ & 18,78 & 0,0000 \\
\hline Modelo polinomial & 0,8377 & 0,8313 & 12,75 & $<0,0001$ & 18,71 & 0,0000 \\
\hline Modelo potencial & 0,9722 & 0,9711 & 12,62 & $<0,0001$ & 18,62 & 0,13831 \\
\hline
\end{tabular}

$R^{2}=$ Coeficiente de determinación normal, $R^{2}$-adj = Coeficiente de determinación ajustado, CME = Cuadrado Medio del Error, Pr>F = Probabilidad de P y Sum res = Suma de residuales.

TABLA 6. Valor del estimador, error estándar y límites de confianza del modelo potencial.

\begin{tabular}{lllllc}
\hline Modelo & Parámetro & Estimador & EE & \multicolumn{2}{l}{ Límites de confianza (95\%) } \\
\hline \multirow{2}{*}{ Modelo potencial } & $\beta_{0}$ & 1,2718 & 0,2712 & 0,7275 & 1,8161 \\
\cline { 2 - 6 } & $\beta_{l}$ & 0,7710 & 0,0559 & 0,6590 & 0,8831 \\
\hline
\end{tabular}

tiene la importante ventaja de ser una función de ahusamiento compatible con la ecuación de volumen existente para el área de estudio.

Pompa-García et al. (2011), mencionan que el uso de la altura del tocón como variable independiente mejora la aplicabilidad del modelo usado. Los autores también indican que es imperativo considerar la altura del tocón en los bosques mexicanos, debido a la diversidad de técnicas de cosecha y a las condiciones topográficas del terreno. En adición, cabe destacar que el modelo utilizado en este trabajo cumple con una propiedad importante que consiste en que el diámetro normal es igual al diámetro del tocón cuando la altura de éste equivale a 1,3 m, aunque en la práctica usualmente no alcanza dicha dimensión. Los mismos autores mencionan que la consistencia en estimación de diámetros de distintos tamaños incorpora una ventaja adicional.

El valor de los estimadores de los parámetros, error estándar (EE) y límites de confianza (al 95\%) del mejor modelo potencial elegido se presentan en la tabla 6. El gráfico de dispersión de residuales contra valores predichos, generado con el modelo, se muestra en el inciso b de la figura 2, visualizando la ausencia de varianzas heterogéneas.

\section{Relación volumen-diámetro del tocón ( $V$ - $D t)$}

La gráfica de dispersión de datos del volumen respecto al diámetro del tocón se representa en la figura 3-a, en donde se muestra que la dependencia entre estas variables dasométricas es de tipo no lineal. Al ajustar los modelos de interés a la base de datos, se obtuvieron los indicadores estadísticos que se muestran en la tabla 7.

Los modelos biomatemáticos ajustados explican $98 \%$ de la variabilidad de la información, con una raíz del cuadrado medio del error de 0,23 , un coeficiente de variación aceptable y una suma de residuales cercana a cero (en ambos casos el valor de P fue de 0,0001), por lo que se deduce que se puede estimar de forma confiable el volumen a través del diámetro del tocón con cualquiera de ellos.

Al evaluar la similitud de los indicadores estadísticos entre los modelos ajustados, y para recomendar un modelo en particular, se realiza el análisis siguiente. En la etapa de ajuste, el modelo de Schumacher arrojó el valor más bajo 

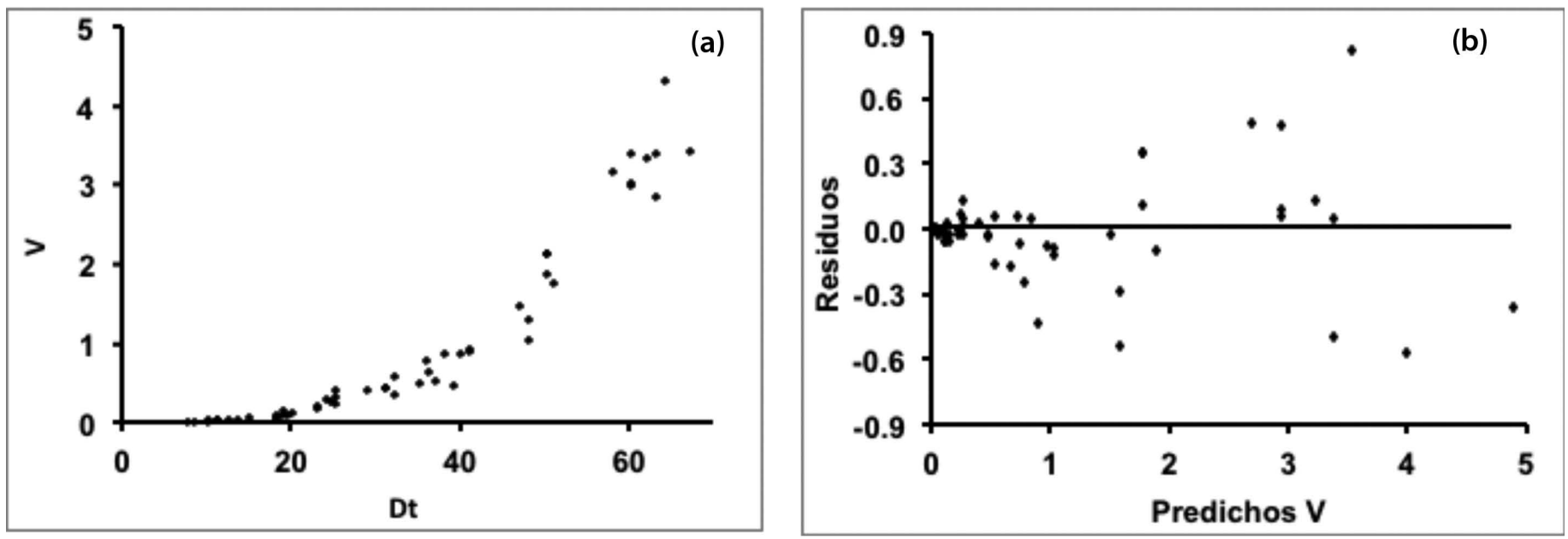

Figura 3. Dispersión de valores del volumen contra diámetro del tocón (a), y dispersión de residuales contra valores predichos de volumen (b), para Quercus laurina en Ixtlán de Juárez, Oaxaca, México.

TABLA 7. Indicadores estadísticos de los modelos ajustados para estimar el volumen respecto al diámetro del tocón para Quercus laurina, en Ixtlán de Juárez, Oaxaca, México.

\begin{tabular}{lllllll}
\hline Modelo & $R^{2}$ & $R^{2}$-adj & $C M E$ & Pr $>F$ & $C V(\%)$ & Sum res \\
\hline Modelo potencial & 0,9805 & 0,9798 & 0,0556 & $<0,0001$ & 22,12 & $-0,6277$ \\
\hline Modelo de Schumacher & 0,9811 & 0,9806 & 0,0534 & $<0,0001$ & 21,67 & 1,9550 \\
\hline
\end{tabular}

$R^{2}=$ Coeficiente de determinación normal, $R^{2}$-adj = Coeficiente de determinación ajustado, CME = Cuadrado Medio del Error, Pr>F = Probabilidad de Py Sum res = Suma de residuales.

en el cuadrado medio del error, lo mismo que para el coeficiente de variación. Sin embargo, al graficar los residuales contra valores predichos, se detecta una ligera tendencia, presencia de heterogeneidad de varianzas en valores menores a $2 \mathrm{~m}^{3}$ de volumen. El modelo potencial generó un cuadrado medio del error y un coeficiente de variación ligeramente superiores al otro modelo, el coeficiente de determinación normal y ajustado son muy similares, pero en la suma de residuos este modelo generó el valor más cercano a cero, además de ausencia de algún tipo de tendencia de valores en la gráfica de residuales contra valores predichos (Fig. 3-b).

Por ello, se concluye que el modelo con mayor confiabilidad para la relación funcional requerida es el modelo potencial. El coeficiente de asimetría y curtosis del modelo potencial fueron cercanos a los valores de la curva de distribución normal.

Siguiendo el criterio de simplicidad para elegir el modelo más apropiado, se concuerda con Barrio et al. (2004), quienes mencionan que la utilización de ecuaciones de cubicación de árbol individual de volumen total demasiado complicadas no aporta nada nuevo a la explicación de la variabilidad del volumen. En su trabajo reducen el abanico de modelos a considerar para la construcción de tarifas de cubicación de volumen total a únicamente 3 modelos. El resultado obtenido en el análisis de la relación funcional de este proyecto, es similar al publicado por Corral-Rivas et al. (2007), quienes mencionan que la relación volumen-diámetro del tocón puede ser modelada de mejor manera a través de una ecuación alométrica. 
TABLA 8. Valor del estimador, error estándar y límites de confianza del modelo potencial.

\begin{tabular}{llllll}
\hline Modelo & Parámetro & Estimador & EE & \multicolumn{2}{c}{ Límites de confianza (95\%) } \\
\hline \multirow{2}{*}{ Modelo potencial } & $\beta_{0}$ & 0,000033 & 0,000016 & 0,0000014 & 0,000065 \\
\cline { 2 - 6 } & $\beta_{1}$ & 2,7812 & 0,1165 & 2,5474 & 3,0149 \\
\hline
\end{tabular}

Los estimadores de los parámetros, sus errores estándar (EE) y límites de confianza $(=0,05)$ del modelo potencial se muestran en la tabla 8 . Con ellos puede obtenerse la ecuación para predecir el volumen respecto al diámetro del tocón para Quercus laurina en Ixtlán de Juárez, Oax.

\section{CONCLUSIONES}

La estimación del diámetro normal a partir del diámetro del tocón puede realizarse de forma confiable por medio de una regresión lineal simple. La altura se puede estimar de forma precisa con base en el diámetro del tocón por medio del modelo potencial. Con el modelo potencial también puede estimarse el volumen a partir del diámetro del tocón.

Las relaciones funcionales generadas son de utilidad para reconstruir escenarios en las áreas afectadas por condiciones naturales o efectos adversos dentro del área de estudio. Esta información es de utilidad para determinar las dimensiones individuales y el volumen total de Quercus laurina en las áreas afectadas, posibilitando también la estimación de biomasa y carbono.

La estimación de variables dasométricas con el uso de modelos simples es una técnica sencilla y de alta confiabilidad para ser empleada en la predicción del crecimiento en masas forestales del estado de Oaxaca, como en el caso de la presente aportación; el uso de modelos complejos debe ser analizado cuidadosamente para determinar si la adición de variables transformadas incrementa la variabilidad explicada por el modelo.

\section{REFERENCIAS}

Aigbe H.I., W.W. Modogu y B.A. Oyebade. 2012. Modeling volume from stump diameter of Terminalia ivorensis (A. CHEV) in Sokponba Forest Reserve, Edo State, Nigeria. ARPN Journal of Agricultural and Biological Science 7(3):146-151.

Alder D. y F. Cailliez. 1980. Estimación del volumen forestal y predicción del rendimiento, con referencia especial a los trópicos. Estudios FAO Montes 22/2. Vol. 2. Roma, Italia. $91 \mathrm{p}$.

Avery, T.E. y H.E. Burkhart. 2002. Forest measurements. 5a ed. McGraw-Hill. Nueva York, EUA. 456 p.

Barrio, A.M., J.G. Álvarez G. e I.J. Díaz-Maroto H. 2004. Elaboración de una tarifa con clasificación de productos para Quercus rubur L. en Galicia, basada en un modelo de volumen porcentual. Investigación agraria. Sistemas y recursos forestales 13(3):506-517.

Benítez N., J.Y., M. Rivero V., A. Vidal C., J. Rodríguez R. y R. C. Álvarez R. 2004. Estimación del diámetro normal a partir del diámetro del tocón en plantaciones de Casuarina equisetifolia Forst. de la provincia Camagüey, Cuba. Revista Chapingo, serie ciencias forestales y del ambiente 10(001):25-30.

Bylin, V.C. 1982. Volume prediction from stump diameter and stump height of selected species in Louisiana. USDA. For. Res. Ser. Pap. SO-182. 11 p.

Corral-Rivas, J.J., M. Barrio A., O. Aguirre C. y U. Diéguez A. 2007. Use of stump diameter to estimate diameter at breast height and tree volume for major pines species in El Salto, Durango, Mexico. Forestry 80(1):29-40.

Cruz C., F., H.M. de los Santos P. y J.R. Valdez L. 2008. Sistema compatible de ahusamiento-volumen para Pinus 
cooperi Blanco en Durango, México. Agrociencia $42(4): 473-485$.

Diéguez A., U., M. Barrio A., F. Castedo D. y M. Balboa M. 2003. Estimación del diámetro normal y del volumen del tronco a partir de las dimensiones del tocón para seis especies forestales comerciales de Galicia. Investigación agraria. Sistemas y recursos forestales 12(2):131-139.

Gujarati D., N. 2004. Econometría. 4a ed. McGraw-Hill Interamericana Editores. México, D.F. 972 p.

Gutiérrez H., P. y R. de la Vara S. 2008. Análisis y diseño de experimentos. McGraw-Hill Companies, Inc. México. 545 p.

Husch B., T.W. Beers y J.A. Kershaw Jr. 2003. Forest mensuration. 4a ed. John Wiley and Sons, Inc. Nueva York. EUA. $443 \mathrm{p}$.

Jenkins J., D. Chojnacky, L. Heath y R. Birdsey. 2004. Comprehensive database of diameter-based biomass regressions for North American tree species. USDA. Gen. Tech. Rep. NE-319. $45 \mathrm{p}$.

McClure, J.P. 1968. Predicting tree dbh from stump measurements in the southeast. USDA For. Ser. Res. Note SE-99. $60 \mathrm{pp}$.

Martínez L., J. 2001. Índice de sitio y comportamiento diamétrico para Pinus patula Schl. et Cham., en San Juan Evangelista Analco, Ixtlán, Oaxaca. Tesis de Maestría. Instituto Tecnológico Agropecuario de Oaxaca núm. 23. Nazareno, Xoxocotlán, Oaxaca. México. 163 p.

Ozcelik, R., J. Brooks, M. Diamentopoulou y H. Wiat. 2010. Estimating breast height diameter and volume from stump diameter for the tree economically important species in Turkey. Scandinavian Journal of Forest Research $25(01): 32-45$

Pacheco F., M. 2011. Tabla de volumen para Quercus laurina en la comunidad de Ixtlán de Juárez, Oaxaca. Tesis profesional. Universidad de la Sierra Juárez. Ixtlán de Juárez, Oaxaca, México. 62 p.

Pompa-García, M., H.M. De los Santos P., M.E. Zepeda-Bautista y J.J. Corral-Rivas. 2011. Un modelo dendrométrico para estimación del diámetro normal a partir de las dimensiones del tocón. Agrociencia 45:379-387.

Pompa-García M. y R. Solís-Moreno. 2008. Ecuación de volumen para el género Quercus en la región noreste de Chi- huahua, México. Revista de Ciencias Forestales Quebracho (16):84-93.

Rentería A., J.B. 1995. Sistema de cubicación para Pinus cooperi blanco mediante ecuaciones de ahusamiento en Durango. Tesis de Maestría en Ciencias. Universidad Autónoma Chapingo. Chapingo, México. 77 p.

Ruiz A., P. 2011. Índice de sitio y predicción de la altura en base al diámetro para Pinus patula Schl. et Cham., en Ixtlán de Juárez, Oaxaca. Tesis profesional. Universidad de la Sierra Juárez. Ixtlán de Juárez, Oaxaca, México. 64 p.

Statistical Analysis System Institute Inc. (SAS). 2002. User's Guide. Version 9.0 for Windows. Cary, N.C. 595 p.

Schlieter, A.J. 1986. Estimation of diameter at breast height from stump diameter for Lodgepole pine. USDA, For. Ser. Res. Note INT-359. 4 p.

Senyurt M. 2012. The relationships between diameter stump height $(\mathrm{d} 0.30)$ and diameter breast height $(\mathrm{d} 1.30)$ for Scotch Pine (Pinus sylvestris L.) in West Black sea Region. Artvin Çoruh Üniversitesi Orman Fakültesi Dergisi 13(1):79-87. http://edergi.artvin.edu.tr

Shrivastava, M.B. y R.A. Singh. 2003. Interrelationships among crown diameter, diameter at breast height and stump diameters of Silver fir trees. VII World Forestry Congress. Quebec, Canadian. http://www.fao.org/DOCREP/ARTICLE/WFC/XII/0902-B4.HTM. Consultado el 30 de mayo de 2012.

Técnica Informática Aplicada S.A. (TIASA). 2003. Programa de manejo forestal para la comunidad de Ixtlán de Juárez, Oax. Oaxaca, México. 231 p.

Westfall, A.J. 2010. New models for predicting diameter at breast height from stump dimensions. Northern Journal of Applied Forestry 27(1):21-27.

Wharton, H.E. 1984. Predicting diameter at breast height from stump diameter for Northeastern tree species. USDA For. Ser. Res. Note NE-322. 4 p.

Williams J.C., A.H. Johnson, B.A. LePage, D.R. Vann y K.D. Taylor. 2003. Reconstruction of tertiary metasequoia forests. I. Test of a method for biomass determination based on stem dimensions. Paleobiology 29 (2):256-270.

Valencia A., S. y K.C. Nixon. 2004. Encinos. In: A.J. GarcíaMendoza, M.J. Ordóñez y M. Briones-Salas, eds. Biodi- 
versidad de Oaxaca. Instituto de Biología, UNAM-Fondo Oaxaqueño para la Conservación de la Naturaleza-World Wildlife Fund, México. p:219-225.

Vanclay, J.K. 1994. Modeling forest growth and yield, applications to mixed tropical forests. CAB International. EUA. 312 p.
Manuscrito recibido el 7 de noviembre de 2012 Aceptado el 12 de noviembre de 2013.

Este documento se debe citar como:

Martínez-López, J. y A. Acosta-Ramos. 2014. Estimación del diámetro, altura y volumen a partir del diámetro del tocón para Quercus laurina, en Ixtlán, Oaxaca, México. Madera y Bosques 20(1):59-70. 